\title{
Editorial: Hierarchical Materials for Advanced Energy Storage
}

\author{
Guanglin Xia ${ }^{1 *}$, Tengfei Zhou ${ }^{2 *}$ and Xuebin $\mathrm{Yu}^{1 *}$ \\ ${ }^{1}$ Department of Materials Science, Fudan University, Shanghai, China, ${ }^{2}$ Institute for Superconducting \& Electronic Materials, \\ University of Wollongong, Wollongong, NSW, Australia
}

Keywords: hierarchical structure, hydrogen storage, electrochemical energy storage, interface, metal hydrides

\section{Editorial on the Research Topic}

\section{Hierarchical Materials for Advanced Energy Storage}

It is urgent to search clean and renewable energy sources for maintaining the economic growth of modern society. In order to realize the versatile, clean, and efficient use of renewable energy, developing efficient energy storage materials and devices is of critical importance. To date, hydrogen storage and electrochemical energy storage are two main types of energy storage systems. Building hierarchical structures has been widely demonstrated to be an effective in advancing various energy storage materials owing to the unique physical and chemical properties induced by tuning their particle sizes, phases, and pores. Hierarchical structures offer several key advantages for advancing hydrogen storage materials and electrochemical energy storage materials, including:

OPEN ACCESS

Edited by:

$\mathrm{Jie} \mathrm{He}$,

University of Connecticut,

United States

Reviewed by:

Sen Zhang,

University of Virginia, United States

*Correspondence:

Guanglin Xia

xiaguanglin@fudan.edu.cn

Tengfei Zhou

tengfeiz@uow.edu.au

Xuebin Yu

yuxuebin@fudan.edu.cn

Specialty section:

This article was submitted to

Nanoscience,

a section of the journal

Frontiers in Chemistry

Received: 02 September 2020

Accepted: 26 October 2020

Published: 12 November 2020

Citation:

Xia G, Zhou T and Yu X (2020)

Editorial: Hierarchical Materials for

Advanced Energy Storage.

Front. Chem. 8:601947.

doi: 10.3389/fchem.2020.601947 (i) the increased surface/contact area per unit mass, which could realize the fast diffusion and adsorption of active species; (ii) the tuned interface structures, which could promote the chemical reaction at the interface toward advanced energy storage performance; (iii) the accommodation of the mechanical strain, which could alleviate the structural damage upon the energy storage process and hence result in superior cycling stability. In this special issue, all the published papers could promote our understanding of the mechanism behind the improvement of energy storage materials via building hierarchical structures.

In this topic, Kim et al. reported the synthesis of binder-free cathode based on Fe foam modified with $\mathrm{FeS}_{2}$ for application in thermal batteries. The work of Zhang et al. demonstrated that, when applied in supercapacitors, three-dimensional core-branch $\alpha-\mathrm{Fe}_{2} \mathrm{O}_{3} @ \mathrm{NiO} / \mathrm{Carbon}$ cloth exhibits high areal capacitance and stable cycling performance due to the reduction of contact resistance and the free-standing structure of the flexible electrode. The flexible and boron-Doped carbon nanotube film built by Wang et al. presents good rate capability and excellent cycling performance when used as a flexible anode in Li ion batteries. Xia et al. demonstrated the potential application of hierarchical structured electrocatalysts for overall water splitting. Moreover, Liu et al. presented a brief review about the progress of electrocatalytic hydrogen production induced by hierarchical porous molybdenum carbide-based nanomaterials and Guan et al. reviewed the progress of advanced supercapacitor materials using two-dimensional transition metal oxide and hydroxide-based hierarchical architectures.

In the field of hydrogen storage, Ali et al. revealed that the catalytic role of $\mathrm{K}_{2} \mathrm{NbF}_{7}$ on the dehydrogenation performance of $\mathrm{LiAlH}_{4}$ could be mainly attributed to in-formed $\mathrm{NbF}_{4}, \mathrm{LiF}$, and $\mathrm{K}$ or K-containing phases during heating for hydrogen storage while Li et al. investigated the influence of various $\mathrm{Al}$ sources on the dehydrogenation behavior of $\mathrm{LiBH}_{4}$. According to the work of Chen et al., when adopting nanoporous Ni-based alloy as templates, the hydrogen storage performance of $\mathrm{LiBH} 4$ could be significantly improved owing to the reduction of particle size. In addition, Sun et al., reviewed the recent progress on improving the hydrogen storage performance of $\mathrm{MgH}_{2}$ using transition metals and carbon materials. 
Finally, as the Guest Editors of this topic issue, we would like to express our gratitude to all the authors for their valuable contributions and all the referees for their hard work and kind help. We hope that this special issue could boost the readers' research interest in building hierarchical structures toward advanced energy storage performance.

\section{AUTHOR CONTRIBUTIONS}

All authors listed have made a substantial, direct and intellectual contribution to the work, and approved it for publication.

\section{FUNDING}

This work was partially supported by the National Key R\&D Program of China (No. 2018YFB1502101), National Science
Fund for Distinguished Young Scholars (51625102), the National Natural Science Foundation of China (51971065 and 51901045), the Innovation Program of Shanghai Municipal Education Commission (2019-01-07-00-07-E00028), and the Programs for Professor of Special Appointment (Eastern Scholar) at Shanghai Institutions of Higher Learning.

Conflict of Interest: The authors declare that the research was conducted in the absence of any commercial or financial relationships that could be construed as a potential conflict of interest.

Copyright (c) $2020 \mathrm{Xia}$, Zhou and Yu. This is an open-access article distributed under the terms of the Creative Commons Attribution License (CC BY). The use, distribution or reproduction in other forums is permitted, provided the original author(s) and the copyright owner(s) are credited and that the original publication in this journal is cited, in accordance with accepted academic practice. No use, distribution or reproduction is permitted which does not comply with these terms. 